\title{
Exact solution of the Izergin-Korepin model with general non-diagonal boundary terms
}

\author{
Kun Hao, ${ }^{a}$ Junpeng Cao, ${ }^{b, c}$ Guang-Liang Li, ${ }^{d}$ Wen-Li Yang, ${ }^{a, e}$ Kangjie Shi ${ }^{a}$ \\ and Yupeng Wang ${ }^{b, c}$ \\ ${ }^{a}$ Institute of Modern Physics, Northwest University, \\ Xian 710069, China \\ ${ }^{b}$ Beijing National Laboratory for Condensed Matter Physics, \\ Institute of Physics, Chinese Academy of Sciences, \\ Beijing 100190, China \\ ${ }^{c}$ Collaborative Innovation Center of Quantum Matter, \\ Beijing, China \\ ${ }^{d}$ Department of Applied Physics, Xian Jiaotong University, \\ Xian 710049, China \\ ${ }^{e}$ Beijing Center for Mathematics and Information Interdisciplinary Sciences, \\ Beijing, 100048, China \\ E-mail: haoke72@163.com, junpengcao@iphy.ac.cn, \\ leegl@mail.xjtu.edu.cn, wlyang@nwu.edu.cn, kjshi@nwu.edu.cn, \\ yupeng@iphy.ac.cn
}

ABSTRACT: The Izergin-Korepin model with general non-diagonal boundary terms, a typical integrable model beyond A-type and without U(1)-symmetry, is studied via the offdiagonal Bethe ansatz method. Based on some intrinsic properties of the $R$-matrix and the $K$-matrices, certain operator product identities of the transfer matrix are obtained at some special points of the spectral parameter. These identities and the asymptotic behaviors of the transfer matrix together allow us to construct the inhomogeneous $T-Q$ relation and the associated Bethe ansatz equations. In the diagonal boundary limit, the reduced results coincide exactly with those obtained via other methods.

KEYwords: Bethe Ansatz, Lattice Integrable Models

ARXIV EPRINT: 1403.7915 


\section{Contents}

1 Introduction 1

2 Transfer matrix 2

3 Operator identities of the transfer matrix $\quad 6$

4 Functional relations and the T-Q relation $\quad 9$

$\begin{array}{llr}4.1 & \text { T-Q ansatz } & 10\end{array}$

4.2 Reduction to the conventional $T-Q$ ansatz 12

$\begin{array}{lll}4.2 .1 & \text { For a generic } \eta & 13\end{array}$

$\begin{array}{lll}\text { 4.2.2 } & \text { For some degenerate } \eta & 14\end{array}$

5 Reduction to the case with diagonal $K$-matrices $\quad 14$

$\begin{array}{llr}6 & \text { Conclusions } & 15\end{array}$

$\begin{array}{ll}\text { A Proof of the crossing symmetry } & 16\end{array}$

\section{Introduction}

Exactly solvable models (or quantum integrable systems) [1] have provided important benchmarks for the non-perturbative analysis of quantum systems appearing in string and super-symmetric Yang-Mills (SYM) theories [2] (see also [3] and references therein), low-dimensional condensed matter physics $[4,5]$ and statistical physics [6-8]. However, it has been well known for many years that there exists a quite usual class of integrable models, which do not possess U(1)-symmetry and thus make the conventional Bethe ansatz methods almost inapplicable. Some famous examples are the closed XYZ chain with odd number of sites [9], the anisotropic spin torus [10], the quantum spin chains with nondiagonal boundary fields [11-15] and their multi-component generalizations [16-21]. The broken $\mathrm{U}(1)$-symmetry in those models leads to the absence of an obvious reference state, which is crucial for the usage of the conventional Bethe ansatz (BA) methods [1, 9, 22-28]. There have been numerous efforts $[6,7,11-15,29-46]$ to approach the exact solutions ${ }^{1}$ to this class of models over the last 20 years.

Very recently, based on the intrinsic properties of the $R$-matrix and the $K$-matrices for quantum integrable models, a systematic method for identifying the spectrum of integrable models without U(1)-symmetry, i.e., the off-diagonal Bethe ansatz (ODBA) method was

\footnotetext{
${ }^{1}$ It is also interesting that the eigenstates can been classified by the representation of the so-called q-Onsager algebra $[47,48]$ and that the half-infinite XXZ spin chain with a triangular boundary has been studied by q-vertex operator method [49].
} 
proposed in [43-46] for the models associated with su(2) algebra. Subsequently, the nestedversion of ODBA for the models associated with $s u(n)$ algebra was developed in [21]. Several long-standing models were then diagonalized exactly $[21,43-46,50,51]$ by the ODBA method. ${ }^{2}$ Despite those progresses, an important issue, i.e., whether the ODBA method can be applied to other multi-component integrable models defined beyond the $s u(n)$ algebra, is still open.

In this paper, we study the Izergin-Korepin (IK) model [57] with generic integrable boundaries. This model has played a fundamental role in quantum integrable models associated with algebras beyond $s u(n)$ (or non A-type models). It was introduced as a quantum integrable model related to the Dodd-Bullough-Mikhailov or Jiber-MikhailovShabat model $[58,59]$, one of two integrable relativistic models containing one scalar field (the other is sine-Gordon model). The $R$-matrix of the model corresponds to the simplest twisted affine algebra $A_{2}^{(2)}$. The IK model with open boundary condition is related to the loop models [60] and self-avoiding walks at a boundary [61]. The IK model with $\mathrm{U}(1)$-symmetry, i.e. with periodic boundary condition or with diagonal boundaries have been extensively studied [60,62-72]. Even the most general integrable boundary condition (corresponding to the non-diagonal reflection matrix) has been known almost for 20 years $[64,69,71]$, its exact solution is still missing. The purpose of this paper is to propose the spectrum of this model using the ODBA method.

The paper is organized as follows. Section 2 serves as an introduction to the model and our notations. In section 3 , we derive certain operator product identities for the transfer matrix of the model with general non-diagonal boundary terms by using some intrinsic properties of the $R$-matrix and $K$-matrices. The asymptotic behaviors of the transfer matrix are also obtained. Section 4 is devoted to the construction of the inhomogeneous $T-Q$ relation and the corresponding Bethe ansatz equations. Section 5 is attributed to the reduction to case of diagonal boundaries. It is found that the reduced results coincide exactly with those obtained by other Bethe ansatz methods. In section 6 , we summarize our results and give some discussions. Some detailed technical proof is given in appendix A.

\section{Transfer matrix}

Throughout, $\mathbf{V}$ denotes a three-dimensional linear space and let $\{|i\rangle \mid i=1,2,3\}$ be an orthonormal basis of it. We shall adopt the standard notations: for any matrix $A \in$ $\operatorname{End}(\mathbf{V}), A_{j}$ is an embedding operator in the tensor space $\mathbf{V} \otimes \mathbf{V} \otimes \cdots$, which acts as $A$ on the $j$-th space and as identity on the other factor spaces; for $B \in \operatorname{End}(\mathbf{V} \otimes \mathbf{V}), B_{i j}$ is an embedding operator of $B$ in the tensor space, which acts as identity on the factor spaces except for the $i$-th and $j$-th ones.

\footnotetext{
${ }^{2}$ The completeness of the solutions for the (an-)isotropic spin- $\frac{1}{2}$ chain has been checked numerically within the Bethe ansatz method $[52,53]$, and holds by construction in the quantum separation variables method (SOV) $[54,55]$ or the q-Onsager algebra method [36]. An expression for the corresponding eigenvectors for the XXX open chain in the framework of algebraic Bethe ansatz was also proposed recently in [56].
} 
The $R$-matrix $R(u) \in \operatorname{End}(\mathbf{V} \otimes \mathbf{V})$ of the IK model is given by [57]

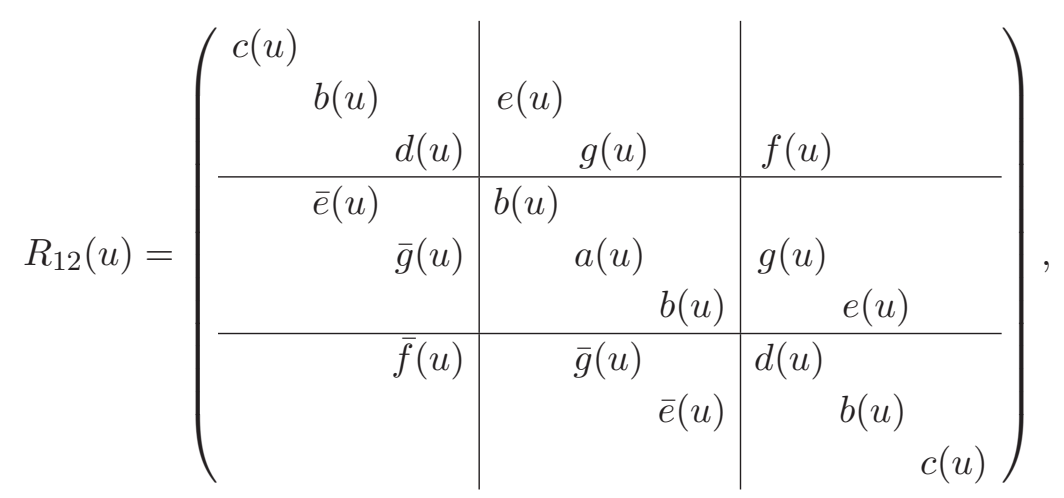

where the matrix elements are

$$
\begin{array}{rlrl}
a(u) & =\sinh (u-3 \eta)-\sinh 5 \eta+\sinh 3 \eta+\sinh \eta, & b(u) & =\sinh (u-3 \eta)+\sin 3 \eta, \\
c(u) & =\sinh (u-5 \eta)+\sinh \eta, & d(u)=\sinh (u-\eta)+\sinh \eta, \\
e(u) & =-2 e^{-\frac{u}{2}} \sinh 2 \eta \cosh \left(\frac{u}{2}-3 \eta\right), & \bar{e}(u) & =-2 e^{\frac{u}{2}} \sinh 2 \eta \cosh \left(\frac{u}{2}-3 \eta\right), \\
f(u) & =-2 e^{-u+2 \eta} \sinh \eta \sinh 2 \eta-e^{-\eta} \sinh 4 \eta, & & \\
\bar{f}(u) & =2 e^{u-2 \eta} \sinh \eta \sinh 2 \eta-e^{\eta} \sinh 4 \eta, & & \bar{g}(u)=-2 e^{\frac{u}{2}-2 \eta} \sinh \frac{u}{2} \sinh 2 \eta . \\
g(u) & =2 e^{-\frac{u}{2}+2 \eta} \sinh \frac{u}{2} \sinh 2 \eta, &
\end{array}
$$

The $R$-matrix satisfies the quantum Yang-Baxter equation (QYBE)

$$
R_{12}\left(u_{1}-u_{2}\right) R_{13}\left(u_{1}-u_{3}\right) R_{23}\left(u_{2}-u_{3}\right)=R_{23}\left(u_{2}-u_{3}\right) R_{13}\left(u_{1}-u_{3}\right) R_{12}\left(u_{1}-u_{2}\right),
$$

and possesses the following properties,

$$
\begin{aligned}
\text { Initial condition : } & R_{12}(0) & =(\sinh \eta-\sinh 5 \eta) P_{12}, \\
\text { Unitarity relation : } & R_{12}(u) R_{21}(-u) & =\rho_{1}(u) \times \mathrm{id}, \\
\text { Crossing relation : } & R_{12}(u) & =V_{1} R_{12}^{t_{2}}(-u+6 \eta+i \pi) V_{1}^{-1}, \\
\text { PT-symmetry : } & R_{21}(u) & =R_{12}^{t_{1} t_{2}}(u), \\
\text { Periodicity : } & R_{12}(u+2 i \pi) & =R_{12}(u) .
\end{aligned}
$$

Here $R_{21}(u)=P_{12} R_{12}(u) P_{12}$ with $P_{12}$ being the usual permutation operator and $t_{i}$ denotes transposition in the $i$-th space. The function $\rho_{1}(u)$ and the crossing matrix $V$ are given by

$$
\begin{aligned}
\rho_{1}(u) & =-4 \sinh \left(\frac{u}{2}-2 \eta\right) \sinh \left(\frac{u}{2}+2 \eta\right) \cosh \left(\frac{u}{2}-3 \eta\right) \cosh \left(\frac{u}{2}+3 \eta\right), \\
V & =\left(\begin{array}{c}
-e^{-\eta} \\
1 \\
-e^{\eta}
\end{array}\right), \quad V^{2}=1 .
\end{aligned}
$$

The unitarity property (2.5) and crossing relation (2.6) of the $R$-matrix and expressions (2.9)-(2.10) of the function $\rho_{1}(u)$ and the $V$-matrix imply that the $R$-matrix satisfies 
the crossing-unitarity relation

$$
\begin{aligned}
& R_{12}^{t_{1}}(u) \mathcal{M}_{1} R_{21}^{t_{1}}(-u+12 \eta) \mathcal{M}_{1}^{-1}=\rho_{2}(u) \times \mathrm{id}, \\
& \mathcal{M}=V^{t} V=\left(\begin{array}{ccc}
e^{2 \eta} & & \\
& 1 & \\
& & e^{-2 \eta}
\end{array}\right), \\
& \rho_{2}(u)=-4 \cosh \left(\frac{u}{2}-5 \eta\right) \cosh \left(\frac{u}{2}-\eta\right) \sinh \frac{u}{2} \sinh \left(\frac{u}{2}-6 \eta\right) .
\end{aligned}
$$

It is easily to check that the $R$-matrix also satisfies the following relation

$$
\mathcal{M}_{1} \mathcal{M}_{2} R_{12}(u) \mathcal{M}_{1}^{-1} \mathcal{M}_{2}^{-1}=R_{12}(u), \quad R_{12}(u) R_{21}(v)=R_{21}(v) R_{12}(u) .
$$

Let us introduce the "row-to-row" (or one-row) monodromy matrices $T(u)$ and $\hat{T}(u)$, which are $3 \times 3$ matrices with operator-valued elements acting on $\mathbf{V}^{\otimes N}$,

$$
\begin{aligned}
& T_{0}(u)=R_{0 N}\left(u-\theta_{N}\right) R_{0 N-1}\left(u-\theta_{N-1}\right) \cdots R_{01}\left(u-\theta_{1}\right), \\
& \hat{T}_{0}(u)=R_{10}\left(u+\theta_{1}\right) R_{20}\left(u+\theta_{2}\right) \cdots R_{N 0}\left(u+\theta_{N}\right) .
\end{aligned}
$$

Here $\left\{\theta_{j} \mid j=1, \cdots, N\right\}$ are arbitrary free complex parameters which are usually called as inhomogeneous parameters. The transfer matrix can be constructed as follows [28, 73]. Let us introduce further a pair of $K$-matrices $K^{-}(u)$ and $K^{+}(u)$. The former satisfies the reflection equation $(\mathrm{RE})$

$$
\begin{aligned}
& R_{12}\left(u_{1}-u_{2}\right) K_{1}^{-}\left(u_{1}\right) R_{21}\left(u_{1}+u_{2}\right) K_{2}^{-}\left(u_{2}\right) \\
& \quad=K_{2}^{-}\left(u_{2}\right) R_{12}\left(u_{1}+u_{2}\right) K_{1}^{-}\left(u_{1}\right) R_{21}\left(u_{1}-u_{2}\right),
\end{aligned}
$$

and the latter satisfies the dual RE

$$
\begin{aligned}
& R_{12}\left(u_{2}-u_{1}\right) K_{1}^{+}\left(u_{1}\right) \mathcal{M}_{1}^{-1} R_{21}\left(-u_{1}-u_{2}+12 \eta\right) \mathcal{M}_{1} K_{2}^{+}\left(u_{2}\right) \\
& \quad=K_{2}^{+}\left(u_{2}\right) \mathcal{M}_{2}^{-1} R_{12}\left(-u_{1}-u_{2}+12 \eta\right) \mathcal{M}_{2} K_{1}^{+}\left(u_{1}\right) R_{21}\left(u_{2}-u_{1}\right) .
\end{aligned}
$$

For open boundaries, one needs to consider the double-row monodromy matrix $\mathbb{T}(u)$

$$
\mathbb{T}(u)=T(u) K^{-}(u) \hat{T}(u) .
$$

The double-row transfer matrix $t(u)$ is thus given by

$$
t(u)=\operatorname{tr}\left(K^{+}(u) \mathbb{T}(u)\right) .
$$

The QYBE (2.3) and (dual) REs (2.17) and (2.18) lead to the fact that the transfer matrices with different spectral parameters commute with each other $[28]:[t(u), t(v)]=$ 0 . Therefore $t(u)$ serves as the generating functional of the conserved quantities of the corresponding system.

In this paper we consider the generic non-diagonal $K$-matrix $K^{-}(u)$ found in [64] and was classified as type $\mathrm{II}^{3}$ in $[69,71]$

$$
K^{-}(u)=\left(\begin{array}{ccc}
1+2 e^{-u-\epsilon} \sinh \eta & 0 & 2 e^{-\epsilon+\sigma} \sinh u \\
0 & 1-2 e^{-\epsilon} \sinh (u-\eta) & 0 \\
2 e^{-\epsilon-\sigma} \sinh u & 0 & 1+2 e^{u-\epsilon} \sinh \eta
\end{array}\right)
$$

\footnotetext{
${ }^{3}$ The generalization to the non-diagonal $K$-matrices of type I in $[69,71]$ is straightforward.
} 
and non-diagonal $K^{+}(u)$ given by

$$
K^{+}(u)=\left.\mathcal{M} K^{-}(-u+6 \eta+i \pi)\right|_{(\epsilon, \sigma) \rightarrow\left(\epsilon^{\prime}, \sigma^{\prime}\right)} .
$$

Besides the crossing parameter $\eta$, the corresponding transfer matrix $t(u)$ given by $(2.20)$ has four other free parameters $\left\{\epsilon, \sigma, \epsilon^{\prime}, \sigma^{\prime}\right\}$ describing the boundary fields. The Hamiltonian of the Izergin-Korepin model with general non-diagonal boundary terms specified by the $K$-matrices given by (2.21) and (2.22) then is given in terms of the transfer matrix by

$$
\begin{aligned}
& H=\left.\frac{\partial \ln t(u)}{\partial u}\right|_{u=0,\left\{\theta_{j}=0\right\}} \\
& =\sum_{j=1}^{N-1} \frac{2 P_{j j+1} R_{j j+1}^{\prime}(0)}{\sinh \eta-\sinh 5 \eta}+\frac{\operatorname{tr} K^{+^{\prime}}(0)}{\operatorname{tr} K^{+}(0)}+\frac{2 t r_{0}\left[K_{0}^{+}(0) P_{N 0} R_{N 0}^{\prime}(0)\right]}{(\sinh \eta-\sinh 5 \eta) t r K^{+}(0)}+\frac{K_{1}^{-\prime}(0)}{1+2 e^{-\epsilon} \sinh \eta} \\
& =\frac{2}{\sinh \eta-\sinh 5 \eta} \sum_{j=1}^{N-1}\left\{\cosh 5 \eta\left(E_{j}^{11} E_{j+1}^{11}+E_{j}^{33} E_{j+1}^{33}\right)\right. \\
& +\sinh 2 \eta(\sinh 3 \eta-\cosh 3 \eta)\left(E_{j}^{11} E_{j+1}^{22}+E_{j}^{22} E_{j+1}^{33}\right) \\
& +\sinh 2 \eta(\sinh 3 \eta+\cosh 3 \eta)\left(E_{j}^{22} E_{j+1}^{11}+E_{j}^{33} E_{j+1}^{22}\right) \\
& +2 \sinh \eta \sinh 2 \eta\left(e^{-2 \eta} E_{j}^{11} E_{j+1}^{33}+e^{2 \eta} E_{j}^{33} E_{j+1}^{11}\right)+\cosh \eta\left(E_{j}^{13} E_{j+1}^{31}+E_{j}^{31} E_{j+1}^{13}\right) \\
& +\cosh 3 \eta\left(E_{j}^{12} E_{j+1}^{21}+E_{j}^{21} E_{j+1}^{12}+E_{j}^{22} E_{j+1}^{22}+E_{j}^{23} E_{j+1}^{32}+E_{j}^{32} E_{j+1}^{23}\right) \\
& \left.-e^{-2 \eta} \sinh 2 \eta\left(E_{j}^{12} E_{j+1}^{32}+E_{j}^{21} E_{j+1}^{23}\right)+e^{2 \eta} \sinh 2 \eta\left(E_{j}^{23} E_{j+1}^{21}+E_{j}^{32} E_{j+1}^{12}\right)\right\} \\
& -\frac{2 e^{-\epsilon}}{1+2 e^{-\epsilon} \sinh \eta}\left[\sinh \eta\left(E_{1}^{11}-E_{1}^{33}\right)+\cosh \eta E_{1}^{22}-e^{\sigma} E_{1}^{13}-e^{-\sigma} E_{1}^{31}\right] \\
& +2\left[(\sinh \eta-\sinh 5 \eta)\left(2 \cosh 2 \eta-4 e^{-\epsilon^{\prime}} \sinh \eta \cosh 4 \eta+1+2 e^{-\epsilon^{\prime}} \sinh 5 \eta\right)\right]^{-1} \\
& \times\left\{\left[\left(e^{2 \eta}-2 e^{-4 \eta-\epsilon^{\prime}} \sinh \eta\right) \cosh 5 \eta+\left(1+2 e^{-\epsilon^{\prime}} \sinh 5 \eta\right)\right.\right. \\
& \left.\times \sinh 2 \eta(\sinh 3 \eta-\cosh 3 \eta)+2\left(e^{-4 \eta}-2 e^{2 \eta-\epsilon^{\prime}} \sinh \eta\right) \sinh \eta \sinh 2 \eta\right] E_{N}^{11} \\
& +\left[\left(e^{2 \eta}-2 e^{-4 \eta-\epsilon^{\prime}} \sinh \eta\right) \sinh 2 \eta(\sinh 3 \eta+\cosh 3 \eta)+\left(1+2 e^{-\epsilon^{\prime}} \sinh 5 \eta\right)\right. \\
& \left.\times \cosh 3 \eta+2\left(e^{-2 \eta}-2 e^{4 \eta-\epsilon^{\prime}} \sinh \eta\right) \sinh 2 \eta(\sinh 3 \eta-\cosh 3 \eta)\right] E_{N}^{22} \\
& +\left[2\left(e^{4 \eta}-2 e^{-2 \eta-\epsilon^{\prime}} \sinh \eta\right) \sinh \eta \sinh 2 \eta+\left(1+2 e^{-\epsilon^{\prime}} \sinh 5 \eta\right)\right. \\
& \left.\times \sinh 2 \eta(\sinh 3 \eta+\cosh 3 \eta)+\left(e^{-2 \eta}-2 e^{4 \eta-\epsilon^{\prime}} \sinh \eta\right) \cosh 5 \eta\right] E_{N}^{33} \\
& \left.-2 e^{-\epsilon^{\prime}} \sinh 6 \eta \cosh \eta\left[e^{2 \eta+\sigma^{\prime}} E_{N}^{13}+e^{-2 \eta-\sigma^{\prime}} E_{N}^{31}\right]\right\} \\
& +\frac{2 e^{-\epsilon^{\prime}}(2 \sinh \eta \sinh 4 \eta-\cosh 5 \eta)}{2 \cosh 2 \eta-4 e^{-\epsilon^{\prime}} \sinh \eta \cosh 4 \eta+1+2 e^{-\epsilon^{\prime}} \sinh 5 \eta},
\end{aligned}
$$

where $E_{j}^{\mu \nu}$ is the Weyl matrix or the Hubbard operator

$$
E^{\mu \nu}=|\mu\rangle\langle\nu|,
$$


and

$$
R_{i j}^{\prime}(u)=\frac{\partial}{\partial u} R_{i j}^{\prime}(u), \quad K^{ \pm \prime}(u)=\frac{\partial}{\partial u} K_{j}^{ \pm}(u)
$$

\section{Operator identities of the transfer matrix}

Following [21] we apply the fusion technique to study the the present model. In this case, we need to use the fusion techniques both for $R$-matrices [74-78] and for $K$-matrices [66, 79]. Similar as that in [21], the fusion procedure will lead to the desired operator identities to determine the spectrum of the transfer matrix $t(u)$ given by $(2.20)$. For this purpose, let us introduce the following vectors in the tensor space $\mathbf{V} \otimes \mathbf{V}$

$$
\begin{aligned}
\left|\Phi_{0}\right\rangle & =\frac{1}{\sqrt{2 \cosh 2 \eta+1}}\left(e^{-\eta}|1,3\rangle-|2,2\rangle+e^{\eta}|3,1\rangle\right), \\
\left|\Phi_{1}\right\rangle & =\frac{1}{\sqrt{2 \cosh 2 \eta}}\left(e^{-\eta}|1,2\rangle-e^{\eta}|2,1\rangle\right), \\
\left|\Phi_{2}\right\rangle & =\frac{1}{\sqrt{2 \cosh 2 \eta}}(|1,3\rangle-2 \sinh \eta|2,2\rangle-|3,1\rangle), \\
\left|\Phi_{3}\right\rangle & =\frac{1}{\sqrt{2 \cosh 2 \eta}}\left(e^{-\eta}|2,3\rangle-e^{\eta}|3,2\rangle\right),
\end{aligned}
$$

and the associated projectors ${ }^{4}$

$$
P_{12}^{(1)}=\left|\Phi_{0}\right\rangle\left\langle\Phi_{0}\left|, \quad P_{12}^{(3)}=\sum_{i=1}^{3}\right| \Phi_{i}\right\rangle\left\langle\Phi_{i}\right| .
$$

Direct calculation shows that the $R$-matrix given by (2.1) at some degenerate points is proportional to the projectors,

$$
R_{12}(6 \eta+i \pi)=P_{12}^{(1)} \times S_{12}^{(1)}, \quad R_{12}(4 \eta)=P_{12}^{(3)} \times S_{12}^{(3)},
$$

where $S_{12}^{(i)}$ are some non-degenerate matrices $\in \operatorname{End}(\mathbf{V} \otimes \mathbf{V})$. After some calculations, we find

$$
\begin{aligned}
P_{12}^{(1)} R_{23}(u) R_{13}(u+6 \eta+i \pi) P_{12}^{(1)} & =\rho_{1}(u) P_{12}^{(1)} \otimes \mathrm{id}=\operatorname{Det}_{q}(R(u)) P_{12}^{(1)} \otimes \mathrm{id}, \\
P_{12}^{(1)} R_{31}(u) R_{32}(u+6 \eta+i \pi) P_{12}^{(1)} & =\rho_{1}(u) P_{12}^{(1)} \otimes \mathrm{id}, \\
P_{12}^{(3)} R_{23}(u) R_{13}(u+4 \eta) P_{12}^{(3)} & =\rho_{3}(u) R_{13}(u+2 \eta+i \pi), \\
P_{12}^{(3)} R_{31}(u) R_{32}(u+4 \eta) P_{12}^{(3)} & =\rho_{3}(u) R_{31}(u+2 \eta+i \pi),
\end{aligned}
$$

where the function $\rho_{3}(u)$ is

$$
\rho_{3}(u)=-2 \sinh \left(\frac{u}{2}+2 \eta\right) \cosh \left(\frac{u}{2}-3 \eta\right) .
$$

Noting that the rank of the projector $P_{12}^{(1)}$ is one, the quantum determinant [80] $\operatorname{Det}_{q}(R(u))$ is defined by

$$
\operatorname{Det}_{q}(R(u))=\operatorname{tr}_{12}\left\{P_{12}^{(1)} R_{23}(u) R_{13}(u+6 \eta+i \pi) P_{12}^{(1)}\right\},
$$

\footnotetext{
${ }^{4}$ In contrast to most of rational models, here $P_{12}^{(i)} \neq P_{21}^{(i)}$.
} 
which plays the role of the generating function of the centers of the associated quantum algebras [81]. In our particular case the corresponding quantum determinant is proportional to the identity operator,

$$
\operatorname{Det}_{q}(R(u))=\rho_{1}(u) \text { id. }
$$

The above relations (3.7)-(3.13) imply that the associated one-row monodromy matrices satisfy the following relations

$$
\begin{aligned}
P_{12}^{(1)} T_{2}(u) T_{1}(u+6 \eta+i \pi) P_{12}^{(1)} & =\prod_{l=1}^{N} \rho_{1}\left(u-\theta_{l}\right) P_{12}^{(1)} \otimes \mathrm{id}, \\
P_{12}^{(1)} \hat{T}_{1}(u) \hat{T}_{2}(u+6 \eta+i \pi) P_{12}^{(1)} & =\prod_{l=1}^{N} \rho_{1}\left(u+\theta_{l}\right) P_{12}^{(1)} \otimes \mathrm{id}, \\
P_{12}^{(3)} T_{2}(u) T_{1}(u+4 \eta) P_{12}^{(1)} & =\prod_{l=1}^{N} \rho_{3}\left(u-\theta_{l}\right) T_{1}(u+2 \eta+i \pi), \\
P_{12}^{(3)} \hat{T}_{1}(u) \hat{T}_{2}(u+4 \eta) P_{12}^{(1)} & =\prod_{l=1}^{N} \rho_{3}\left(u+\theta_{l}\right) \hat{T}_{1}(u+2 \eta+i \pi),
\end{aligned}
$$

where $\left\{\theta_{l} \mid l=1, \ldots, N\right\}$ are the inhomogeneous parameters defined in (2.15)-(2.16).

Now let us construct the corresponding fusion procedures for the $K$-matrices following $[66,79]$. The properties (3.6) of the $R$-matrix at the degenerate points and the RE $(2.17)$ and its dual RE (2.18) allow us to construct the fused $K$-matrices from the origin ones (2.21) and (2.22). After some tedious calculations, we find that

$$
\begin{aligned}
P_{21}^{(1)} K_{1}^{-}(u) R_{21}(2 u+6 \eta+i \pi) K_{2}^{-}(u+6 \eta+i \pi) P_{12}^{(1)} & =\operatorname{Det}_{q}\left(K^{-}(u)\right) P_{21}^{(1)}, \\
P_{12}^{(1)} K_{2}^{+}(u+6 \eta+i \pi) \mathcal{M}_{1} R_{12}(-2 u+6 \eta+i \pi) \mathcal{M}_{1}^{-1} K_{1}^{+}(u) P_{21}^{(1)} & =\operatorname{Det}_{q}\left(K^{+}(u)\right) P_{12}^{(1)}, \\
P_{21}^{(3)} K_{1}^{-}(u) R_{21}(2 u+4 \eta) K_{2}^{-}(u+4 \eta) P_{12}^{(3)} & =f_{-}(u) K_{1}^{-}(u+2 \eta+i \pi), \\
P_{12}^{(3)} K_{2}^{+}(u+4 \eta) \mathcal{M}_{1} R_{12}(-2 u+8 \eta) \mathcal{M}_{1}^{-1} K_{1}^{+}(u) P_{21}^{(3)} & =f_{+}(u) K_{1}^{+}(u+2 \eta+i \pi),
\end{aligned}
$$

where the functions $f_{ \pm}(u)$ and $\operatorname{Det}_{q}\left(K^{ \pm}(u)\right)$ are

$$
\begin{aligned}
f_{-}(u)= & -2\left(1-2 e^{-\epsilon} \sinh (u-\eta)\right) \cosh (u-\eta) \sinh (u+4 \eta) \\
f_{+}(u)= & 2\left(1-2 e^{-\epsilon^{\prime}} \sinh (u-\eta)\right) \cosh (u-\eta) \sinh (u-6 \eta) \\
\operatorname{Det}_{q}\left(K^{-}(u)\right)= & \operatorname{tr}_{12}\left\{P_{21}^{(1)} K_{1}^{-}(u) R_{21}(2 u+6 \eta+i \pi) K_{2}^{-}(u+6 \eta+i \pi) P_{12}^{(1)}\right\} \\
= & -2\left(1-2 e^{-\epsilon} \sinh (u-\eta)\right)\left(1+e^{-\epsilon} \sinh (u+\eta)\right) \\
& \times \sinh (u+6 \eta) \cosh (u+\eta), \\
\operatorname{Det}_{q}\left(K^{+}(u)\right)= & \operatorname{tr}_{12}\left\{P_{12}^{(1)} K_{2}^{+}(u+6 \eta+i \pi) \mathcal{M}_{1} R_{12}(-2 u+6 \eta+i \pi) \mathcal{M}_{1}^{-1} K_{1}^{+}(u) P_{21}^{(1)}\right\} \\
= & 2\left(1-2 e^{-\epsilon^{\prime}} \sinh (u-\eta)\right)\left(1+e^{-\epsilon^{\prime}} \sinh (u+\eta)\right) \\
& \times \sinh (u-6 \eta) \cosh (u-\eta) .
\end{aligned}
$$


Following the method in [21] and using the relations (3.14)-(3.21), after a long calculation, we find the transfer matrix given by (2.20) satisfies the following operator identities

$$
\begin{aligned}
t\left( \pm \theta_{j}\right) t\left( \pm \theta_{j}+6 \eta+i \pi\right) & =\left.\frac{\delta_{1}(u) \times \mathrm{id}}{\rho_{1}(2 u)}\right|_{u= \pm \theta_{j}}, & j=1, \ldots, N \\
t\left( \pm \theta_{j}\right) t\left( \pm \theta_{j}+4 \eta\right) & =\left.\frac{\delta_{2}(u) \times t(u+2 \eta+i \pi)}{\rho_{2}(-2 u+8 \eta)}\right|_{u= \pm \theta_{j}}, & j=1, \ldots, N .
\end{aligned}
$$

Here the functions $\delta_{1}(u)$ and $\delta_{2}(u)$ are

$$
\begin{aligned}
& \delta_{1}(u)=\operatorname{Det}_{q}\left(K^{-}(u)\right) \operatorname{Det}_{q}\left(K^{+}(u)\right) \prod_{l=1}^{N} \rho_{1}\left(u-\theta_{l}\right) \rho_{1}\left(u+\theta_{l}\right), \\
& \delta_{2}(u)=f_{-}(u) f_{+}(u) \prod_{l=1}^{N} \rho_{3}\left(u-\theta_{l}\right) \rho_{3}\left(u+\theta_{l}\right) .
\end{aligned}
$$

Following the method in [82] and using the the explicit expressions (2.21) and (2.22) of the $K$-matrices, we find that the transfer matrix (2.20) possesses the crossing symmetry

$$
t(u)=t(-u+6 \eta+i \pi)
$$

The proof of the above relation is given in appendix A. We remark that the very operator identities (3.26)-(3.27) at the points $-\theta_{j}$ is guaranteed by their equalities at the points $\theta_{j}$ and the crossing symmetry (3.30) of the transfer matrix. Therefore, in the following we will use the operator identities (3.26)-(3.27) at the points $\theta_{j}$ and the crossing symmetry (3.30) to determine the eigenvalues of the transfer matrix.

Now let us derive some properties of the transfer matrix. Firstly the expressions of the $K$-matrices $K^{ \pm}(u)$ given by $(2.21)$ and (2.22) imply that $K^{ \pm}(u)$ have the following periodicity

$$
K^{ \pm}(u+2 i \pi)=K^{ \pm}(u) .
$$

This leads to the periodicity of the transfer matrix $t(u)$

$$
t(u+2 i \pi)=t(u)
$$

Moreover the explicit expressions of the $R$-matrix and the $K$-matrices allow us to have the following asymptotic behaviors of the transfer matrix

$$
\lim _{u \rightarrow \pm \infty} t(u)=\left(\frac{1}{2}\right)^{2 N} e^{-\epsilon-\epsilon^{\prime}} e^{ \pm 2(N+1)(u-3 \eta)}\left(1+2 \cosh \left(\sigma^{\prime}-\sigma+2 \eta\right)\right) \times \mathrm{id}+\ldots
$$

The explicit expressions of the $K$-matrices given by (2.21) and (2.22) also imply that $K^{ \pm}(u)$ satisfy the following properties

$$
\begin{aligned}
K^{-}(0) & =\left(1+2 e^{-\epsilon} \sinh \eta\right), & K^{+}(6 \eta+i \pi) & =\left(1+2 e^{-\epsilon^{\prime}} \sinh \eta\right) \mathcal{M}, \\
K^{-}(i \pi) & =\left(1-2 e^{-\epsilon} \sinh \eta\right), & K^{+}(6 \eta) & =\left(1-2 e^{-\epsilon^{\prime}} \sinh \eta\right) \mathcal{M} .
\end{aligned}
$$


The unitarity (2.5) and the crossing unitarity (2.11) of the $R$-matrix imply that the one-row monodromy matrices $T(u)$ given by $(2.15)$ and $\hat{T}(u)$ given by $(2.16)$ satisfy the following relations

$$
\begin{array}{r}
T_{0}(u) \hat{T}_{0}(-u)=\prod_{l=1}^{N} \rho_{1}\left(u-\theta_{l}\right) \times \mathrm{id}, \\
T_{0}^{t_{0}}(u) \mathcal{M}_{0} \hat{T}_{0}^{t_{0}}(-u+12 \eta) \mathcal{M}_{0}^{-1}=\prod_{l=1}^{N} \rho_{2}\left(u-\theta_{l}\right) \times \mathrm{id} .
\end{array}
$$

The above relations allow us to work out the transfer matrix at special points $0, i \pi, 6 \eta, 6 \eta+$ $i \pi$ as follows

$$
\begin{gathered}
t(0)=t(6 \eta+i \pi)=\left(1+2 e^{-\epsilon} \sinh \eta\right) \operatorname{tr}\left\{K^{+}(0)\right\} \prod_{l=1}^{N} \rho_{1}\left(-\theta_{l}\right) \times \mathrm{id}, \\
t(i \pi)=t(6 \eta)=\left(1-2 e^{-\epsilon} \sinh \eta\right) \operatorname{tr}\left\{K^{+}(i \pi)\right\} \prod_{l=1}^{N} \rho_{1}\left(i \pi-\theta_{l}\right) \times \mathrm{id} .
\end{gathered}
$$

\section{Functional relations and the T-Q relation}

The property $[t(u), t(v)]=0$ implies that the eigenstates of $t(u)$ are independent of $u$. Suppose $|\Psi\rangle$ is an eigenstate of $t(u)$ with an eigenvalue $\Lambda(u)$, namely,

$$
t(u)|\Psi\rangle=\Lambda(u)|\Psi\rangle
$$

The very operator identities (3.26)-(3.27) of the transfer matrix at the points $\theta_{j}$ imply the corresponding eigenvalue $\Lambda(u)$ satisfies the similar relations

$$
\begin{aligned}
\Lambda\left(\theta_{j}\right) \Lambda\left(\theta_{j}+6 \eta+i \pi\right) & =\left.\frac{\delta_{1}(u)}{\rho_{1}(2 u)}\right|_{u=\theta_{j}}, & j=1, \ldots, N, \\
\Lambda\left(\theta_{j}\right) \Lambda\left(\theta_{j}+4 \eta\right) & =\left.\frac{\delta_{2}(u) \times \Lambda(u+2 \eta+i \pi)}{\rho_{2}(-2 u+8 \eta)}\right|_{u=\theta_{j}}, & j=1, \ldots, N,
\end{aligned}
$$

where the functions $\delta_{i}(u)$ are given by (3.28) and (3.29). The crossing symmetry (3.30) of the transfer matrix leads to the crossing symmetry of its eigenvalues

$$
\Lambda(u)=\Lambda(-u+6 \eta+i \pi) .
$$

The properties of the transfer matrix $t(u)$ given by (3.31), (3.32) and (3.37)-(3.38) imply that the corresponding eigenvalue $\Lambda(u)$ satisfies the following relations:

$$
\begin{aligned}
\Lambda(u+2 i \pi) & =\Lambda(u) \\
\Lambda(0) & =\left(1+2 e^{-\epsilon} \sinh \eta\right) \operatorname{tr}\left\{K^{+}(0)\right\} \prod_{l=1}^{N} \rho_{1}\left(-\theta_{l}\right) \\
\Lambda(i \pi) & =\left(1-2 e^{-\epsilon} \sinh \eta\right) \operatorname{tr}\left\{K^{+}(i \pi)\right\} \prod_{l=1}^{N} \rho_{1}\left(i \pi-\theta_{l}\right), \\
\lim _{u \rightarrow \pm \infty} \Lambda(u) & =\left(\frac{1}{2}\right)^{2 N} e^{-\epsilon-\epsilon^{\prime}} e^{ \pm 2(N+1)(u-3 \eta)}\left(1+2 \cosh \left(\sigma^{\prime}-\sigma+2 \eta\right)\right)+\ldots
\end{aligned}
$$


The periodicity (4.4) and the asymptotic behavior (4.7) of the eigenvalue $\Lambda(u)$, the analyticity of the R-matrix and K-matrices and the $u$-free eigenstate lead to the fact that the eigenvalue $\Lambda(u)$ further possesses the property

$\Lambda(u)$, as an entire function of $u$, is a trigonometric polynomial of degree $2 N+2$.

Namely, $\Lambda(u)$ is a Laurent polynomial of $e^{u}$ with $4(N+1)+1$ unknown coefficients. The crossing relation (4.3) reduces the number of the independent unknown coefficients to $2(N+1)+1$. Thus one needs $2(N+1)+1$ conditions to determine these coefficients. Therefore the relations (4.1)-(4.8) completely characterize the spectrum of the transfer matrix of the model.

\subsection{T-Q ansatz}

Based on the properties (4.1)-(4.8) of the eigenvalues $\Lambda(u)$, following the ODBA method developed in [21, 43-46], let us propose the following conjecture for the eigenvalues,

$$
\begin{aligned}
& \Lambda(u)=\prod_{l=1}^{N} c\left(u-\theta_{l}\right) c\left(u+\theta_{l}\right)\left(1-2 e^{-\epsilon} \sinh (u-\eta)\right)\left(1-2 e^{-\epsilon^{\prime}} \sinh (u-\eta)\right) \\
& \times \frac{\sinh (u-6 \eta) \cosh (u-\eta)}{\sinh (u-2 \eta) \cosh (u-3 \eta)} \frac{Q_{1}(u+4 \eta)}{Q_{2}(u)} \\
& +\prod_{l=1}^{N} d\left(u-\theta_{l}\right) d\left(u+\theta_{l}\right)\left(1-2 e^{-\epsilon} \sinh (u-5 \eta)\right)\left(1-2 e^{-\epsilon^{\prime}} \sinh (u-5 \eta)\right) \\
& \times \frac{\sinh u \cosh (u-5 \eta)}{\sinh (u-4 \eta) \cosh (u-3 \eta)} \frac{Q_{2}(u-6 \eta+i \pi)}{Q_{1}(u-2 \eta+i \pi)} \\
& +\prod_{l=1}^{N} b\left(u-\theta_{l}\right) b\left(u+\theta_{l}\right)\left(1+2 e^{-\epsilon} \sinh (u-3 \eta)\right)\left(1+2 e^{-\epsilon^{\prime}} \sinh (u-3 \eta)\right) \\
& \times \frac{\sinh u \sinh (u-6 \eta)}{\sinh (u-2 \eta) \sinh (u-4 \eta)} \frac{Q_{1}(u+2 \eta+i \pi) Q_{2}(u-4 \eta)}{Q_{2}(u-2 \eta+i \pi) Q_{1}(u)} \\
& +4^{1-N} c \frac{\sinh u \sinh (u-6 \eta)}{\cosh (u-3 \eta)} \prod_{l=1}^{N} c\left(u-\theta_{l}\right) c\left(u+\theta_{l}\right) d\left(u-\theta_{l}\right) d\left(u+\theta_{l}\right) \\
& \times\left[\frac{Q_{1}(u+2 \eta+i \pi) \sinh ^{l_{1}}(u-3 \eta) \sinh ^{l_{1}}(u-\eta) \cosh ^{l_{2}}(u-2 \eta)}{Q_{1}(u) Q_{2}(u)}\right. \\
& \left.-(-1)^{l_{2}} \frac{Q_{2}(u-4 \eta) \sinh ^{l_{1}}(u-3 \eta) \sinh ^{l_{1}}(u-5 \eta) \cosh ^{l_{2}}(u-4 \eta)}{Q_{1}(u-2 \eta+i \pi) Q_{2}(u-2 \eta+i \pi)}\right],
\end{aligned}
$$


where the functions $c(u), b(u)$ and $d(u)$ are the elements of the $R$-matrix given by $(2.2)$ and the functions $Q_{i}(u)$ and the constant $c$ are given by

$$
\begin{aligned}
& Q_{1}(u)=\prod_{k=1}^{\bar{N}} \sinh \left[\frac{u-\lambda_{k}}{2}-\eta\right], \quad \bar{N}=4\left(N+l_{1}\right)+2\left(l_{2}-1\right), \\
& Q_{2}(u)=\prod_{k=1}^{\bar{N}} \sinh \left[\frac{u+\lambda_{k}}{2}-\eta\right] \\
& c=(-1)^{\left(l_{2}-1\right)} e^{-\epsilon-\epsilon^{\prime}}\left\{\frac{\cosh \left(\sigma^{\prime}-\sigma+2 \eta\right)-\cosh \left(\delta_{l_{1}, l_{2}} \eta-\sum_{j=1}^{\bar{N}} \lambda_{j}\right)}{\cosh \left(\frac{\delta_{l_{1}, l_{2}} \eta}{2}-\frac{1}{2} \sum_{j=1}^{\bar{N}} \lambda_{j}\right)}\right\}, \\
& \delta_{l_{1}, l_{2}}=4 N+8 l_{1}+4 l_{2}-2 .
\end{aligned}
$$

Here $l_{1}$ and $l_{2}$ are arbitrary non-negative integers. ${ }^{5}$ It is easy to check that the functions $Q_{i}(u)$ possess the following properties

$$
Q_{i}(u+2 i \pi)=Q_{i}(u), \quad \text { for } i=1,2, \quad \text { and } \quad Q_{2}(u)=Q_{1}(-u+4 \eta) .
$$

With the help of the above relations, we have checked that the $T-Q$ ansatz (4.9) indeed satisfies the relations (4.3)-(4.6). Moreover the special choice of the constant $c$ given by (4.12) implies that the ansatz also satisfies the asymptotic behavior (4.7). The explicit expressions of the functions $b(u), c(u)$ and $d(u)$ given by (2.1)-(2.2) imply that

$$
b(0)=d(0)=c(6 \eta+i \pi)=b(6 \eta+i \pi)=c(4 \eta)=d(2 \eta+i \pi)=0 .
$$

These properties give rise to the fact that the $T-Q$ ansatz (4.9) satisfies the very functional relations (4.1)-(4.2). This means that our ansatz (4.9) actually satisfies the relations (4.1)(4.7) except the analytic properties (4.8).

From the explicit expression (4.9) of $\Lambda(u)$, one may find that the $T-Q$ ansatz (4.9) might have some apparent simple poles at the following points:

$$
2 \eta, 4 \eta, 3 \eta+i \frac{\pi}{2}, \quad \bmod (i \pi)
$$

and

$$
\lambda_{j}+2 \eta,-\lambda_{j}+2 \eta, \lambda_{j}+4 \eta+i \pi,-\lambda_{j}+4 \eta+i \pi, \quad \bmod (2 i \pi), \quad j=1, \ldots, \bar{N} .
$$

Direct calculation shows that the residues of the $T-Q$ ansatz (4.9) at the points given by (4.16) vanishes, or the ansatz has no singularity at these points. Moreover we have checked that the $T-Q$ ansatz (4.9) also has no singularity at the points given by (4.17)

\footnotetext{
${ }^{5}$ The results of anisotropic spin- $\frac{1}{2}$ chains [43-46] strongly suggest that fixed $l_{1}$ and $l_{2}$ might give a complete set of eigenvalues of the transfer matrix. In such a sense, different $l_{1}$ and $l_{2}$ might only give different parameterizations of the eigenvalues but not different states.
} 
provided that the $\bar{N}$ parameters $\left\{\lambda_{j} \mid j=1, \ldots, \bar{N}\right\}$ satisfy the following BAEs

$$
\begin{aligned}
& \frac{\left(1+2 e^{-\epsilon} \sinh \left(\lambda_{j}-\eta\right)\right)\left(1+2 e^{-\epsilon^{\prime}} \sinh \left(\lambda_{j}-\eta\right)\right) \cosh \left(\lambda_{j}-\eta\right) Q_{2}\left(\lambda_{j}-2 \eta\right) Q_{2}\left(\lambda_{j}+2 \eta\right)}{4 \sinh \lambda_{j} \sinh \left(\lambda_{j}-2 \eta\right) \sinh ^{l_{1}}\left(\lambda_{j}+\eta\right) \sinh ^{l_{1}}\left(\lambda_{j}-\eta\right) \cosh ^{l_{2}} \lambda_{j}} \\
& \quad=-c Q_{2}\left(\lambda_{j}+i \pi\right) \prod_{l=1}^{N} \sinh \left[\frac{\lambda_{j}-\theta_{l}}{2}-\eta\right] \sinh \left[\frac{\lambda_{j}+\theta_{l}}{2}-\eta\right] \cosh \left[\frac{\lambda_{j}-\theta_{l}}{2}\right] \cosh \left[\frac{\lambda_{j}+\theta_{l}}{2}\right], \\
& j=1, \ldots, \bar{N},
\end{aligned}
$$

where the function $Q_{2}(u)$ is given by (4.11). The non-singular property of the $T-Q$ ansatz (4.9) at the points (4.17) can be verified by directly calculating the residues of the ansatz at these points.

Finally we conclude that the $T-Q$ ansatz (4.9) indeed satisfies (4.1)-(4.8) as it is required, if the the $\bar{N}$ parameters $\left\{\lambda_{j} \mid j=1, \ldots, \bar{N}\right\}$ satisfy the associated BAEs (4.18). Thus the $\Lambda(u)$ given by (4.9), when the $\bar{N}$ parameters $\left\{\lambda_{j} \mid j=1, \ldots, \bar{N}\right\}$ satisfy the associated BAEs (4.18), becomes the eigenvalue of the transfer matrix $t(u)$ given by $(2.20)$ for the Izergin-Korepin model with the general non-diagonal boundary terms specified by the non-diagonal $K$-matrices $K^{ \pm}(u)$ given by $(2.21)$ and (2.22).

\subsection{Reduction to the conventional $T-Q$ ansatz}

It follows from (4.12) that the parameter $c$ does depend on not only the boundary parameters but also the parameters $\left\{\lambda_{j}\right\}$ (such a dependence also appeared in the anisotropic spin- $\frac{1}{2}$ chains with arbitrary boundary fields [43-46]). The vanishing condition of $c$, i.e. $c=0$, will lead to the constraint (see (4.23) below) among the boundary parameters, in this case one might find a proper "local vacuum" to apply the conventional Bethe ansatz $[15,83]$. The Bethe ansatz equations (4.18) imply that for this case the parameters $\left\{\lambda_{j}\right\}$ have to form two types of pairs:

$$
\left(\lambda_{j},-\lambda_{j}\right), \quad\left(\lambda_{j},-\lambda_{j}+4 \eta\right)
$$

Suppose the number of the first type pairs is $M$ ( $M$ being a non-negative integer such that $\left.0 \leq M \leq \frac{\bar{N}}{2}\right)$, the resulting $T-Q$ relation (4.9) becomes the conventional one [1]

$$
\begin{aligned}
\Lambda(u)= & \prod_{l=1}^{N} c\left(u-\theta_{l}\right) c\left(u+\theta_{l}\right)\left(1-2 e^{-\epsilon} \sinh (u-\eta)\right)\left(1-2 e^{-\epsilon^{\prime}} \sinh (u-\eta)\right) \\
& \times \frac{\sinh (u-6 \eta) \cosh (u-\eta)}{\sinh (u-2 \eta) \cosh (u-3 \eta)} \frac{Q(u+4 \eta)}{Q(u)} \\
+ & \prod_{l=1}^{N} d\left(u-\theta_{l}\right) d\left(u+\theta_{l}\right)\left(1-2 e^{-\epsilon} \sinh (u-5 \eta)\right)\left(1-2 e^{-\epsilon^{\prime}} \sinh (u-5 \eta)\right) \\
& \times \frac{\sinh u \cosh (u-5 \eta)}{\sinh (u-4 \eta) \cosh (u-3 \eta)} \frac{Q(u-6 \eta-i \pi)}{Q(u-2 \eta+i \pi)} \\
+ & \prod_{l=1}^{N} b\left(u-\theta_{l}\right) b\left(u+\theta_{l}\right)\left(1+2 e^{-\epsilon} \sinh (u-3 \eta)\right)\left(1+2 e^{-\epsilon^{\prime}} \sinh (u-3 \eta)\right) \\
& \times \frac{\sinh u \sinh (u-6 \eta)}{\sinh (u-2 \eta) \sinh (u-4 \eta)} \frac{Q(u+2 \eta+i \pi) Q(u-4 \eta)}{Q(u-2 \eta+i \pi) Q(u)}
\end{aligned}
$$


where the resulting function $Q(u)$ is

$$
Q(u)=\prod_{j=1}^{M} \sinh \left[\frac{u-\lambda_{j}}{2}-\eta\right] \sinh \left[\frac{u+\lambda_{j}}{2}-\eta\right] .
$$

The resulting BAEs become

$$
\begin{aligned}
& \prod_{l=1}^{N} \frac{\sinh \left[\frac{\lambda_{j}-\theta_{l}}{2}-\eta\right] \sinh \left[\frac{\lambda_{j}+\theta_{l}}{2}-\eta\right]}{\sinh \left[\frac{\lambda_{j}-\theta_{l}}{2}+\eta\right] \sinh \left[\frac{\lambda_{j}+\theta_{l}}{2}+\eta\right]} \frac{\left(1-2 e^{-\epsilon} \sinh \left(\lambda_{j}+\eta\right)\right)\left(1-2 e^{-\epsilon^{\prime}} \sinh \left(\lambda_{j}+\eta\right)\right)}{\left(1+2 e^{-\epsilon} \sinh \left(\lambda_{j}-\eta\right)\right)\left(1+2 e^{-\epsilon^{\prime}} \sinh \left(\lambda_{j}-\eta\right)\right)} \\
& \quad=-\frac{\sinh \left(\lambda_{j}+2 \eta\right) \cosh \left(\lambda_{j}-\eta\right)}{\sinh \left(\lambda_{j}-2 \eta\right) \cosh \left(\lambda_{j}+\eta\right)} \frac{Q\left(-\lambda_{j}-2 \eta\right) Q\left(-\lambda_{j}+4 \eta+i \pi\right)}{Q\left(\lambda_{j}-2 \eta\right) Q\left(\lambda_{j}+4 \eta+i \pi\right)}, j=1, \ldots, M .
\end{aligned}
$$

On the other hand, the form of the pairs (4.19) implies that

$$
\sum_{j+1}^{\bar{N}} \lambda_{j}=\sum_{j=1}^{\frac{\bar{N}-2 M}{2}}\left(\lambda_{j}-\lambda_{j}+4 \eta\right)=(2 \bar{N}-4 M) \eta .
$$

The relation (4.12) and the definition (4.10) of $\bar{N}$ give rise to the following constraint between the boundary parameters ${ }^{6}$

$$
\cosh \left(\sigma^{\prime}-\sigma+2 \eta\right)=\cosh (4 M \eta-4 N \eta+2 \eta), \quad 0 \leq M .
$$

\subsubsection{For a generic $\eta$}

For a generic $\eta$, there exist three solutions to the constraint (4.23). If the boundary parameters $\sigma$ and $\sigma^{\prime}$ satisfy the constraint

$$
\sigma^{\prime}-\sigma=-4 k \eta \bmod (2 i \pi), \quad k \leq-N, \quad \text { and } k \in Z,
$$

the corresponding $M$ can take only one allowed value,

$$
M=N-k .
$$

If the boundary parameters $\sigma$ and $\sigma^{\prime}$ satisfy the following constraint

$$
\sigma^{\prime}-\sigma=-4 k \eta \bmod (2 i \pi), \quad 1-N \leq k \leq N, \quad \text { and } k \in Z,
$$

then $M$ can take the two allowed values denoted by $M^{ \pm}$respectively,

$$
M^{-}=N-k, \quad M^{+}=N+k-1 .
$$

If the boundary parameters $\sigma$ and $\sigma^{\prime}$ satisfy

$$
\sigma^{\prime}-\sigma=-4 k \eta \bmod (2 i \pi), N+1 \leq k, \quad \text { and } k \in Z,
$$

\footnotetext{
${ }^{6}$ One can always choose the non-negative integers $l_{1}$ and $l_{2}$ such that $M$ becomes any non-negative integer since that $0 \leq M \leq 2\left(N+l_{1}\right)+l_{2}-1=\bar{N} / 2$.
} 
$M$ can take only one allowed value,

$$
M=N+k-1 .
$$

For the case that the boundary parameters satisfy the relation (4.24) (or (4.28)), the eigenvalue of the transfer matrix is characterized by a fixed $M$ in (4.25) (or (4.29)). This $\Lambda(u)$ itself might give the complete set of the eigenvalues of the transfer matrix. However, if the boundary parameters obey the relation (4.26), there exist two $\Lambda^{ \pm}(u)$ corresponding to the two different allowed $M$ in (4.27). Similar as that of the anisotropic spin- $\frac{1}{2}$ chain with arbitrary boundary fields $[15,37,84,85]$, these two $\Lambda^{ \pm}(u)$ together might constitute the complete set of the eigenvalues of the transfer matrix.

\subsubsection{For some degenerate $\eta$}

Similar as that in the closed XYZ chain and the anisotropic spin- $\frac{1}{2}$ chain with arbitrary boundary fields [43-46], if the isotropic (or crossing) parameter $\eta$ takes the following discrete value

$$
\eta=\frac{\sigma-\sigma^{\prime}}{4 N-4 M}+\frac{2 i \pi m}{4 N-4 M}, \quad m, M \in Z, \quad \text { and } 0 \leq M,
$$

the constraint (4.23) is automatically satisfied for arbitrary boundary parameters $\epsilon, \epsilon^{\prime}, \sigma$ and $\sigma^{\prime}$. Thus in this case the eigenvalue of the transfer matrix is given by (4.20) and the corresponding BAEs are given by (4.22). It should be emphasized that these degenerate points (4.30) become dense in the thermodynamic limit $(N, m, M \rightarrow \infty)$. This enables one to obtain the thermodynamic properties (up to the order of $O\left(N^{-2}\right)$ ) of the model for generic values of $\eta$ via the conventional thermodynamic Bethe ansatz methods [86, 87]. This method has been proven to be very successful in the derivation of the surface energy of the XXZ spin chain with arbitrary boundary fields [88].

\section{Reduction to the case with diagonal $K$-matrices}

Now let us consider the diagonal $K$-matrices (i.e., taking the limits $\epsilon, \epsilon^{\prime} \rightarrow+\infty$ of $(2.21)$ and (2.22)). The resulting $K$-matrices read

$$
K^{-}(u)=\mathrm{id}, \quad K^{+}(u)=\mathcal{M},
$$

where the matrix $\mathcal{M}$ is given by (2.12). The $T-Q$ ansatz (4.20) is reduced to the one [66] obtained by analytic Bethe ansatz method

$$
\begin{aligned}
\Lambda(u)= & \prod_{l=1}^{N} c\left(u-\theta_{l}\right) c\left(u+\theta_{l}\right) \frac{\sinh (u-6 \eta) \cosh (u-\eta)}{\sinh (u-2 \eta) \cosh (u-3 \eta)} \frac{Q(u+4 \eta)}{Q(u)} \\
& +\prod_{l=1}^{N} d\left(u-\theta_{l}\right) d\left(u+\theta_{l}\right) \frac{\sinh u \cosh (u-5 \eta)}{\sinh (u-4 \eta) \cosh (u-3 \eta)} \frac{Q(u-6 \eta+i \pi)}{Q(u-2 \eta+i \pi)} \\
& +\prod_{l=1}^{N} b\left(u-\theta_{l}\right) b\left(u+\theta_{l}\right) \frac{\sinh u \sinh (u-6 \eta)}{\sinh (u-2 \eta) \sinh (u-4 \eta)} \\
& \times \frac{Q(u-4 \eta) Q(u+2 \eta+i \pi)}{Q(u-2 \eta+i \pi) Q(u)}
\end{aligned}
$$


where the function $Q(u)$ is still given by (4.21). In particular, in this case the vanishing condition of $c$ is automatically satisfied thanks to the relation (4.12). In this case the $\mathrm{U}(1)$-symmetry is recovered and $M$ can take any of the following values, ${ }^{7}$

$$
M=0,1, \ldots, 2 N .
$$

For each of the above allowed values, the resulting BAEs become

$$
\begin{gathered}
\prod_{l=1}^{N} \frac{\sinh \left[\frac{\lambda_{j}-\theta_{l}}{2}-\eta\right] \sinh \left[\frac{\lambda_{j}+\theta_{l}}{2}-\eta\right] \sinh \left[\frac{\lambda_{j}-\theta_{l}}{2}+\eta\right] \sinh \left[\frac{\lambda_{j}+\theta_{l}}{2}+\eta\right]}{\sinh \left(\lambda_{j}-2 \eta\right) \cosh \left(\lambda_{j}+\eta\right)} \\
=-\frac{Q\left(-\lambda_{j}-2 \eta\right) Q\left(-\lambda_{j}+4 \eta+i \pi\right)}{Q\left(\lambda_{j}-2 \eta\right) Q\left(\lambda_{j}+4 \eta+i \pi\right)}, \quad j=1, \ldots, M .
\end{gathered}
$$

\section{Conclusions}

The Izergin-Korepin model with general non-diagonal boundary terms specified by the most general non-diagonal $K$ matrices given by (2.21) and (2.22) has been studied by the off-diagonal Bethe ansatz method. Based on some intrinsic properties of the $R$-matrix and $K$-matrices, we derive the very functional relations (3.26) and (3.27) of the transfer matrix. These relations, together with other properties, allow us to construct an off-diagonal (or inhomogeneous) $T-Q$ relation (4.9) of the eigenvalue of the transfer matrix and the associated BAEs (4.18). When the boundary parameters satisfy one constraint (4.23), the resulting $T-Q$ relation is reduced to the conventional one (4.20), which might allow one to use the method developed in [88] to study the thermodynamic properties (up to the order of $O\left(N^{-2}\right)$ ) of the model for generic values of $\eta$ via the conventional thermodynamic Bethe ansatz methods [86, 87]. Taking the limit $\epsilon, \epsilon^{\prime} \rightarrow+\infty$, the corresponding $K$-matrices become diagonal ones and the resulting $T-Q$ relation is then reduced to that in [66].

\section{Acknowledgments}

The financial support from the National Natural Science Foundation of China (Grant Nos. 11174335, 11031005, 11375141, 11374334), the National Program for Basic Research of MOST (973 project under grant No.2011CB921700), the State Education Ministry of China (Grant No. 20116101110017) and BCMIIS are gratefully acknowledged.

\footnotetext{
${ }^{7}$ The allowed values (5.3) of $M$ can be deduced by studying the asymptotic behavior of the eigenvalues of transfer matrix [66].
} 


\section{A Proof of the crossing symmetry}

Direct calculation shows that the $K$-matrices given by (2.21)-(2.22) satisfy the following relations

$$
\begin{aligned}
K^{-}(u) K^{-}(-u) & =\Delta_{-}(u) \times \mathrm{id}, \\
V^{t} K^{+}(-u+6 \eta+i \pi) V V^{t} K^{+}(u+6 \eta+i \pi) V & =\Delta_{+}(u) \times \mathrm{id},
\end{aligned}
$$

where the matrix $V$ is given by $(2.10)$ and the functions $\Delta_{ \pm}(u)$ are

$$
\begin{aligned}
& \Delta_{-}(u)=\left(1-2 e^{-\epsilon} \sinh (u-\eta)\right)\left(1+2 e^{-\epsilon} \sinh (u+\eta)\right), \\
& \Delta_{+}(u)=\left(1-2 e^{-\epsilon^{\prime}} \sinh (u-\eta)\right)\left(1+2 e^{-\epsilon^{\prime}} \sinh (u+\eta)\right) .
\end{aligned}
$$

Similar as those in [82], let us introduce the following matrices $\bar{K}^{ \pm}(u)$

$$
\begin{aligned}
\bar{K}_{1}^{-}(u) & =\operatorname{tr}_{2}\left\{P_{12} R_{21}(-2 u) V_{2} K_{2}^{-t_{2}}(u+6 \eta+i \pi) V_{2}^{t_{2}}\right\}, \\
\bar{K}_{1}^{+}(u+6 \eta+i \pi) & =\operatorname{tr}_{2}\left\{P_{12} R_{12}(2 u) K_{2}^{+t_{2}}(u)\right\} .
\end{aligned}
$$

Substituting the expressions (2.21)-(2.22) of the $K$-matrices into the above relations, we have that the $K$-matrices satisfy the following crossing relation

$$
\begin{aligned}
\bar{K}^{-}(u) & =\frac{\operatorname{Det}_{q}\left(K^{-}(u)\right)}{\Delta_{-}(u)} K^{-}(-u), \\
\bar{K}^{+}(u+6 \eta+i \pi) & =\frac{\operatorname{Det}_{q}\left(K^{+}(u)\right)}{\Delta_{+}(u)} V^{t} K^{+}(-u+6 \eta+i \pi) V,
\end{aligned}
$$

where the functions $\operatorname{Det}\left(K^{-}(u)\right)$ and $\operatorname{Det}\left(K^{+}(u)\right)$ are given respectively by (3.24) and (3.25). Combining the crossing relation (2.6) and PT-symmetry (2.7) of the $R$-matrix, one may derive the following relations

$$
\begin{aligned}
& R_{21}(u)=V_{1}^{t_{1}} R_{12}^{t_{1}}(-u+6 \eta+i \pi) V_{1}^{t_{1}}, \\
& R_{21}(u)=V_{2} R_{12}^{t_{2}}(-u+6 \eta+i \pi) V_{2} .
\end{aligned}
$$

Then the above relations, (2.6) and the expression (2.10) give rise to the following relation between the one-row monodromy matrices $T(u)$ and $\hat{T}(u)$ give by $(2.15)$ and $(2.16)$

$$
\begin{aligned}
& T_{0}^{t_{0}}(-u+6 \eta+i \pi)=V_{0}^{t_{0}} \hat{T}_{0}(u) V_{0}^{t_{0}}, \\
& \hat{T}_{0}^{t_{0}}(-u+6 \eta+i \pi)=V_{0} T_{0}(u) V_{0} .
\end{aligned}
$$

Following the method in [82] and using the QYBE (2.3), we can prove that the transfer matrix specified by the $R$-matrix given by (2.1) and the $K$-matrices given by (2.21) and (2.22) satisfies the crossing symmetry (3.30).

Open Access. This article is distributed under the terms of the Creative Commons Attribution License (CC-BY 4.0), which permits any use, distribution and reproduction in any medium, provided the original author(s) and source are credited. 


\section{References}

[1] R.J. Baxter, Exactly Solved Models in Statistical Mechanics, Academic Press, (1982).

[2] L. Dolan, C.R. Nappi and E. Witten, A Relation between approaches to integrability in superconformal Yang-Mills theory, JHEP 10 (2003) 017 [hep-th/0308089] [INSPIRE].

[3] N. Beisert et al., Review of AdS/CFT integrability: an overview, Lett. Math. Phys. 99 (2012) 3 [arXiv:1012.3982] [INSPIRE].

[4] A.A. Zvyagin, Finite Size Effects in Correlated Electron Models: Exact Results, Imperial College Press, London, U.K. (2005).

[5] X.-W. Guan, M.T. Batchelor and C. Lee, Fermi gases in one dimension: From Bethe ansatz to experiments, Rev. Mod. Phys. 85 (2013) 1633.

[6] J. de Gier and F.H.L. Essler, Bethe Ansatz Solution of the Asymmetric Exclusion Process with Open Boundaries, Phys. Rev. Lett. 95 (2005) 240601 [INSPIRE].

[7] J. de Gier and F.H.L. Essler, Exact spectral gaps of the asymmetric exclusion process with open boundaries, J. Stat. Mech. (2006) P12011 [cond-mat/0609645].

[8] J. Sirker, R.G. Pereira and I. Affleck, Diffusion and ballistic transport in one-dimensional quantum systems, Phys. Rev. Lett. 103 (2009) 216602 [arXiv:0906.1978].

[9] L.A. Takhtadzhan and L.D. Faddeev, The quantum method of the inverse problem and the Heisenberg XYZ model, Russ. Math. Sur. 34 (1979) 11.

[10] C.M. Yung and M.T. Batchelor, Exact solution for the spin s XXZ quantum chain with nondiagonal twists, Nucl. Phys. B 446 (1995) 461 [hep-th/9502041] [INSPIRE].

[11] R.I. Nepomechie, Bethe Ansatz solution of the open XX spin chain with nondiagonal boundary terms, J. Phys. A 34 (2001) 9993 [hep-th/0110081] [INSPIRE].

[12] R.I. Nepomechie, Solving the open XXZ spin chain with nondiagonal boundary terms at roots of unity, Nucl. Phys. B 622 (2002) 615 [hep-th/0110116] [INSPIRE].

[13] R.I. Nepomechie, Functional relations and Bethe Ansatz for the XXZ chain, J. Statist. Phys. 111 (2003) 1363 [hep-th/0211001] [INSPIRE].

[14] R.I. Nepomechie, Bethe ansatz solution of the open XXZ chain with nondiagonal boundary terms, J. Phys. A 37 (2004) 433 [hep-th/0304092] [INSPIRE].

[15] J. Cao, H.-Q. Lin, K.-J. Shi and Y. Wang, Exact solution of XXZ spin chain with unparallel boundary fields, Nucl. Phys. B 663 (2003) 487.

[16] W.L. Yang and R. Sasaki, Solution of the dual reflection equation for $A_{n-1}^{(1)}$ SOS model, J. Math. Phys. 45 (2004) 4301 [hep-th/0308118] [INSPIRE].

[17] W.L. Yang and R. Sasaki, Exact solution of $Z(n)$ Belavin model with open boundary condition, Nucl. Phys. B 679 (2004) 495 [hep-th/0308127] [INSPIRE].

[18] W. Galleas and M.J. Martins, Solution of the $S U(N)$ vertex model with non-diagonal open boundaries, Phys. Lett. A 335 (2005) 167 [nlin/0407027].

[19] W.-L. Yang and Y.-Z. Zhang, Non-diagonal solutions of the reflection equation for the trigonometric $A_{n-1}^{(1)}$ vertex model, JHEP 12 (2004) 019 [hep-th/0411160] [INSPIRE].

[20] W.-L. Yang and Y.-Z. Zhang, Exact solution of the $A_{n-1}^{(1)}$ trigonometric vertex model with non-diagonal open boundaries, JHEP 01 (2005) 021 [hep-th/0411190] [INSPIRE]. 
[21] J. Cao, W.-L. Yang, K. Shi and Y. Wang, Nested off-diagonal Bethe ansatz and exact solutions of the $\mathrm{SU}(N)$ spin chain with generic integrable boundaries, JHEP 04 (2014) 143 [arXiv: 1312.4770] [INSPIRE].

[22] H. Bethe, On the theory of metals. 1. Eigenvalues and eigenfunctions for the linear atomic chain, Z. Phys. 71 (1931) 205 [INSPIRE].

[23] F.C. Alcaraz, M.N. Barber, M.T. Batchelor, R.J. Baxter and G.R.W. Quispel, Surface Exponents of the Quantum XXZ, Ashkin-Teller and Potts Models, J. Phys. A 20 (1987) 6397 [inSPIRE].

[24] R.J. Baxter, Eight-Vertex Model in Lattice Statistics, Phys. Rev. Lett. 26 (1971) 832 [INSPIRE].

[25] R.J. Baxter, One-Dimensional Anisotropic Heisenberg Chain, Phys. Rev. Lett. 26 (1971) 834 [INSPIRE].

[26] R.J. Baxter, One-dimensional anisotropic Heisenberg chain, Annals Phys. 70 (1972) 323 [INSPIRE].

[27] E.K. Sklyanin and L.D. Faddeev, Quantum Mechanical Approach to Completely Integrable Field Theory Models, Sov. Phys. Dokl. 23 (1978) 902 [InSPIRE].

[28] E.K. Sklyanin, Boundary Conditions for Integrable Quantum Systems, J. Phys. A 21 (1988) 2375 [inSPIRE].

[29] J. de Gier and P. Pyatov, Bethe ansatz for the Temperley-Lieb loop model with open boundaries, J. Stat. Mech. (2004) P03002 [hep-th/0312235] [INSPIRE].

[30] A. Nichols, V. Rittenberg and J. de Gier, One-boundary Temperley-Lieb algebras in the XXZ and loop models, J. Stat. Mech. (2005) P03003 [cond-mat/0411512] [INSPIRE].

[31] J. de Gier, A. Nichols, P. Pyatov and V. Rittenberg, Magic in the spectra of the XXZ quantum chain with boundaries at $\Delta=0$ and $\Delta=-1 / 2$, Nucl. Phys. B 729 (2005) 387 [hep-th/0505062] [INSPIRE].

[32] W.-L. Yang, Y.-Z. Zhang and M.D. Gould, Exact solution of the XXZ Gaudin model with generic open boundaries, Nucl. Phys. B 698 (2004) 503 [hep-th/0411048] [INSPIRE].

[33] A. Doikou and P.P. Martin, On quantum group symmetry and Bethe ansatz for the asymmetric twin spin chain with integrable boundary, J. Stat. Mech. (2006) P06004 [hep-th/0503019] [INSPIRE].

[34] A. Doikou, The Open XXZ and associated models at $q$ root of unity, J. Stat. Mech. (2006) P09010 [hep-th/0603112] [INSPIRE].

[35] Z. Bajnok, Equivalences between spin models induced by defects, J. Stat. Mech. (2006) P06010 [hep-th/0601107] [INSPIRE].

[36] P. Baseilhac and K. Koizumi, Exact spectrum of the XXZ open spin chain from the q-Onsager algebra representation theory, J. Stat. Mech. (2007) P09006 [hep-th/0703106] [INSPIRE].

[37] W.-L. Yang, R.I. Nepomechie and Y.-Z. Zhang, Q-operator and T-Q relation from the fusion hierarchy, Phys. Lett. B 633 (2006) 664 [hep-th/0511134] [INSPIRE].

[38] W. Galleas, Functional relations from the Yang-Baxter algebra: Eigenvalues of the XXZ model with non-diagonal twisted and open boundary conditions,

Nucl. Phys. B 790 (2008) 524 [INSPIRE]. 
[39] H. Frahm, A. Seel and T. Wirth, Separation of Variables in the open XXX chain, Nucl. Phys. B 802 (2008) 351 [arXiv:0803.1776] [INSPIRE].

[40] G. Niccoli, Non-diagonal open spin-1/2 XXZ quantum chains by separation of variables: Complete spectrum and matrix elements of some quasi-local operators, J. Stat. Mech. (2012) P10025 [arXiv:1206.0646] [INSPIRE].

[41] G. Niccoli, Antiperiodic spin-1/2 XXZ quantum chains by separation of variables: Complete spectrum and form factors, Nucl. Phys. B 870 (2013) 397 [arXiv:1205.4537] [INSPIRE].

[42] G. Niccoli, Antiperiodic dynamical 6-vertex and periodic 8-vertex models I: Complete spectrum by SOV and matrix elements of the identity on separate states,

J. Phys. A 46 (2013) 075003 [arXiv:1207.1928] [inSPIRE].

[43] J. Cao, W. Yang, K. Shi and Y. Wang, Off-diagonal Bethe ansatz and exact solution of a topological spin ring, Phys. Rev. Lett. 111 (2013) 137201 [arXiv:1305.7328] [INSPIRE].

[44] J. Cao, W.-L. Yang, K. Shi and Y. Wang, Off-diagonal Bethe ansatz solution of the XXX spin-chain with arbitrary boundary conditions, Nucl. Phys. B 875 (2013) 152 [arXiv:1306.1742] [INSPIRE].

[45] J. Cao, W.-L. Yang, K.-J. Shi and u. Wang, Spin-1/2 XYZ model revisit: general solutions via off-diagonal Bethe ansatz, arXiv:1307.0280 [INSPIRE].

[46] J. Cao, W.-L. Yang, K. Shi and Y. Wang, Off-diagonal Bethe ansatz solutions of the anisotropic spin- $\frac{1}{2}$ chains with arbitrary boundary fields, Nucl. Phys. B 877 (2013) 152 [arXiv: 1307.2023] [INSPIRE].

[47] P. Baseilhac, The q-deformed analogue of the Onsager algebra: Beyond the Bethe ansatz approach, Nucl. Phys. B 754 (2006) 309 [math-ph/0604036] [INSPIRE].

[48] P. Baseilhac and S. Belliard, The half-infinite XXZ chain in Onsager's approach, Nucl. Phys. B 873 (2013) 550 [arXiv:1211.6304] [INSPIRE].

[49] P. Baseilhac and T. Kojima, Form factors of the half-infinite XXZ spin chain with a triangular boundary, arXiv:1404.0491 [INSPIRE].

[50] Y.-Y. Li, J. Cao, W.-L. Yang, K. Shi and Y. Wang, Exact solution of the one-dimensional Hubbard model with arbitrary boundary magnetic fields, Nucl. Phys. B 879 (2014) 98 [arXiv: 1311.0432] [INSPIRE].

[51] X. Zhang, J. Cao, W.-L. Yang, K. Shi and Y. Wang, Exact solution of the one-dimensional super-symmetric t-J model with unparallel boundary fields, J. Stat. Mech. (2014) P04031 [arXiv: 1312.0376] [INSPIRE].

[52] R.I. Nepomechie, An inhomogeneous T-Q equation for the open XXX chain with general boundary terms: completeness and arbitrary spin, J. Phys. A 46 (2013) 442002 [arXiv: 1307.5049] [INSPIRE].

[53] Y. Jiang, S. Cui, J. Cao, W.-L. Yang and Y. Wang, Completeness and Bethe root distribution of the spin-1/2 Heisenberg chain with arbitrary boundary fields, arXiv:1309.6456 [INSPIRE].

[54] S. Faldella, N. Kitanine and G. Niccoli, Complete spectrum and scalar products for the open spin-1/2 XXZ quantum chains with non-diagonal boundary terms, J. Stat. Mech. (2014) P01011 [arXiv:1307.3960] [INSPIRE]. 
[55] N. Kitanine, J.-M. Maillet and G. Niccoli, Open spin chains with generic integrable boundaries: Baxter equation and Bethe ansatz completeness from separation of variables, J. Stat. Mech. 1405 (2014) P05015 [arXiv:1401.4901] [INSPIRE].

[56] S. Belliard and N. Crampé, Heisenberg XXX Model with General Boundaries: Eigenvectors from Algebraic Bethe Ansatz, SIGMA (2013) 072.

[57] A.G. Izergin and V.E. Korepin, The inverse scattering method approach to the quantum Shabat-Mikhailov model, Commun. Math. Phys. 79 (1981) 303 [InSPIRE].

[58] R.K. Dodd and R.K. Bullough, Polynomial Conserved Densities for the sine-Gordon Equations, Proc. Roy. Soc. Lond. A 352 (1977) 481 [inSPIRE].

[59] A.V. Zhiber and A.B. Shabat, Klein-Gordon equations with a nontrivial group, Sov. Phys. Dokl. 24 (1979) 607 [INSPIRE].

[60] C.M. Yung and M.T. Batchelor, Integrable vertex and loop models on the square lattice with open boundaries via reflection matrices, Nucl. Phys. B 435 (1995) 430 [hep-th/9410042] [INSPIRE].

[61] M.T. Batchelor and C.M. Yung, Exact Results for the Adsorption of a Flexible Self-Avoiding Polymer Chain in Two Dimensions, Phys. Rev. Lett. 74 (1995) 2026 [cond-mat/9410082].

[62] N.Y. Reshetikhin, The functional equation method in the theory of exactly soluble quantum systems, Sov. Phys. JETP 57 (1983) 691.

[63] V.A. Tarasov, Algebraic Bethe ansatz for the Izergin-Korepin R-matrix, Theor. Math. Phys. 76 (1988) 793.

[64] J.D. Kim, Boundary K matrix for the quantum Mikhailov-Shabat model, hep-th/9412192 [INSPIRE].

[65] M.J. Martins, The Exact solution and the finite size behavior of the Osp(1/2) invariant spin chain, Nucl. Phys. B 450 (1995) 768 [hep-th/9502133] [inSPIRE].

[66] L. Mezincescu and R.I. Nepomechie, Analytical Bethe Ansatz for quantum algebra invariant spin chains, Nucl. Phys. B 372 (1992) 597 [hep-th/9110050] [INSPIRE].

[67] H. Fan, Bethe ansatz for the Izergin-Korepin model, Nucl. Phys. B 488 (1997) 409.

[68] B.-Y. Hou, W.-L. Yang and Y.-Z. Zhang, The twisted quantum affine algebra $U_{q}\left(A_{2}^{(2)}\right)$ and correlation functions of the Izergin-Korepin model, Nucl. Phys. B 556 (1999) 485.

[69] A. Lima-Santos, Reflection K-matrices for 19-vertex models, Nucl. Phys. B 558 (1999) 637.

[70] W.-L. Yang and Y.-Z. Zhang, Izergin-Korepin model with a boundary, Nucl. Phys. B 596 (2001) 495.

[71] R.I. Nepomechie, Boundary quantum group generators of type A, Lett. Math. Phys. 62 (2002) 83 [hep-th/0204181] [INSPIRE].

[72] G.-L. Li, K.-J. Shi and R.-H. Yue, The algebraic Bethe ansatz for the Izergin-Korepin model with open boundary conditions, Nucl. Phys. B 670 (2003) 401.

[73] L. Mezincescu and R.I. Nepomechie, Integrability of open spin chains with quantum algebra symmetry, Int. J. Mod. Phys. A 6 (1991) 5231 [Addendum ibid. A 7 (1992) 5657] [hep-th/9206047] [INSPIRE].

[74] M. Karowski, On the Bound State Problem in (1+1)-dimensional Field Theories, Nucl. Phys. B 153 (1979) 244 [inSPIRE]. 
[75] P.P. Kulish, N.Y. Reshetikhin and E.K. Sklyanin, Yang-Baxter Equation and Representation Theory. 1., Lett. Math. Phys. 5 (1981) 393 [inSPIRE].

[76] P.P. Kulish and E.K. Sklyanin, Quantum spectral transform method recent developments, Lect. Notes Phys. 151 (1982) 61.

[77] A.N. Kirillov and N.Y. Reshetikhin, Exact solution of the Heisenberg XXZ model of spin s, J. Sov. Math. 35 (1986) 2627.

[78] A.N. Kirillov and N.Y. Reshetikhin, Exact solution of the integrable XXZ Heisenberg model with arbitrary spin. I. The ground state and the excitation spectrum, J. Phys. A 20 (1987) 1565 [inSPIRE].

[79] Y.-k. Zhou, Row transfer matrix functional relations for Baxter's eight vertex and six vertex models with open boundaries via more general reflection matrices, Nucl. Phys. B 458 (1996) 504 [hep-th/9510095] [INSPIRE].

[80] P.P. Kulish and E.K. Sklyanin, Quantum inverse scattering method and the Heisenberg ferromagnet, Phys. Lett. A 70 (1979) 461 [InSPIRE].

[81] V. Chari and A. Pressley, A Guide to Quantum Groups, Cambridge University Press, Cambridge (1994).

[82] W.-L. Yang and Y.-Z. Zhang, T-Q relation and exact solution for the XYZ chain with general nondiagonal boundary terms, Nucl. Phys. B 744 (2006) 312 [hep-th/0512154] [INSPIRE].

[83] W.-L. Yang and Y.-Z. Zhang, On the second reference state and complete eigenstates of the open XXZ chain, JHEP 04 (2007) 044 [hep-th/0703222] [INSPIRE].

[84] R.I. Nepomechie and F. Ravanini, Completeness of the Bethe ansatz solution of the open XXZ chain with nondiagonal boundary terms, J. Phys. A 36 (2003) 11391 [hep-th/0307095] [INSPIRE].

[85] R.I. Nepomechie and F. Ravanini, Addendum to 'Completeness of the Bethe Ansatz solution of the open XXZ chain with nondiagonal boundary terms', J. Phys. A 37 (2004) 1945.

[86] V.E. Korepin, N.M. Boliubov and A.G. Izergin, Quantum Inverse Scattering Method and Correlation Functions, Cambridge University Press, Cambridge (1993).

[87] M. Takahashi, Thermodynamics of One-Dimensional Solvable Models, Cambridge University Press, Cambridge (1999).

[88] Y.-Y. Li, J. Cao, W.-L. Yang, K. Shi and Y. Wang, Thermodynamic limit and surface energy of the XXZ spin chain with arbitrary boundary fields, Nucl. Phys. B 884 (2014) 17 [arXiv:1401.3045] [INSPIRE]. 